\title{
Predictive Value of Circulating Tumor Cells in Prognosis of Stage III/IV Colorectal Cancer After Oxaliplatin-based First-line Chemotherapy
}

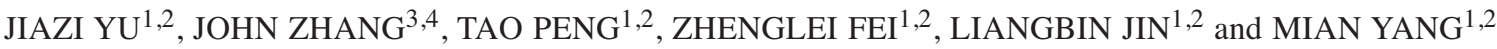 \\ ${ }^{1}$ Department of General Surgery, Ningbo Medical Treatment Centre Li Huili Hospital, Ningbo, P.R. China; \\ ${ }^{2}$ Li Huili Hospital of Ningbo University, Ningbo, P.R. China; \\ ${ }^{3}$ Thayer School of Engineering, Dartmouth College, Engineering Drive, Hanover, NH, U.S.A.; \\ ${ }^{4}$ Norris Cotton Cancer Center, Dartmouth Hitchcock Medical Center, Hanover, NH, U.S.A.
}

\begin{abstract}
Background/Aim: Insufficient data exist to support the concept of the circulating tumor cell (CTC) level as a prognostic factor for platinum-based first-line chemotherapy. This study investigated the impact of CTCs on the prognosis of patients with advanced colorectal cancer (CRC) after receiving platinum-based chemotherapy. Analyses were carried out of clinicopathological features and molecular phenotypes to clarify independent risk factors for a high CTC count. Patients and Methods: Patients diagnosed with stage III/IV CRC ( $n=76)$ were included in the study. The blood samples of patients were evaluated for CTCs using the CellRich ${ }^{T M}$ platform system. Immunohistochemistry (Ias used to analyze epithelial-mesenchymal transition-associated biomarkers E-cadherin and vimentin. Univariate and logistic regression analyses were then conducted to analyze the risk factors for CTC expression. Additionally, the influence of oxaliplatin on disease-free survival after first-line chemotherapy or during chemotherapy was analyzed through a 2-year follow-up. Results: Patients in the $\mathrm{CTC}^{+}$group experienced shorter DFS after receiving oxaliplatin first-line chemotherapy than patients in the $C T C^{-}$group $(p<0.01)$. In addition, univariate analysis revealed that the tumor M-stage, tumor location, RAS mutation, high expression of vimentin, and deletion of E-cadherin expression were correlated with a high CTC count. Multivariate analysis suggested that the
\end{abstract}

This article is freely accessible online.

Correspondence to: Mian Yang, Department of General Surgery, Ningbo Medical Treatment Centre Li Huili Hospital, 1111 Jiangnan road, Ningbo, 315000, P.R. China. Tel: +86 13429364839, e-mail: 939838409@qq.com

Key Words: Circulating tumor cells, colorectal cancer, adjuvant chemotherapy, disease-free survival. presence of RAS gene mutations and high vimentin expression were independent risk factors for high CTC loads $(p<0.01)$. Conclusion: CTC positivity can indicate the efficacy of firstline chemotherapy with oxaliplatin in stage III/IV colorectal cancer. This may be linked to tumor epithelial-mesenchymal transition in patients with CTCs. Moreover, RAS gene mutation and high expression of vimentin were identified as independent risk factors for a high CTC count.

Colorectal carcinoma (CRC) was the second most prevalent cancer and the third most common cause of cancer-related deaths worldwide in 2018 (1). Despite the development of diagnostic and therapeutic modalities in recent years, more than $58 \%$ of patients with advanced CRC have a poor prognosis. Approximately $30 \%$ of patients with CRC experience tumor recurrence and metastasis even after radical resection and adjuvant chemotherapy (2). Potential metastasis and resistance to chemotherapy are the main causes of treatment failure. Therefore, the development of new methods to predict recurrence and metastasis, and that can be used for diagnosis and the evaluation of therapeutic response is of utmost importance for the optimum management of patients with CRC. Also known as 'liquid biopsy', the detection of circulating tumor cells (CTC) is a new diagnostic modality that has been receiving a considerable amount of attention over the past few years (3, 4). Compared with a classic biopsy, it is more convenient, and presents minimal procedural risks to the patient. Over the past 10 years, large-scale clinical studies have focused on the use of CTC counts as predictors of prognosis and the response to therapy, particularly in patients with breast or prostate cancer. Despite the fact that the analysis of published clinical studies provides coherent evidence that the presence of CTCs in the peripheral blood is a strong prognostic factor in patients with CRC, there is insufficient data to determine whether CTC count is also a prognostic 
factor predicting the efficacy of platinum-based first-line chemotherapy.

Several factors promoting high CTC counts have been identified. Epithelial-mesenchymal transition (EMT), a phenomenon common in normal physiological processes and critical during tumor invasion and metastasis, might be one of the most important risk factors for high CTC counts (5). TNM staging, tumor size and other clinicopathological factors may also lead to high CTC counts $(6,7)$. However, the results obtained from previously published studies were inconsistent on the causes of an increased level of CTCs, and did not provide clinicopathological features and commonly used molecular phenotypes to determine the actual risk factors for this.

In this study, we analyzed the relationship between presence of CTCs and the prognosis of patients with stage III/IV CRC after platinum-based first-line chemotherapy. To the best of our knowledge, this study is the first to combine univariate and multivariate analyses to demonstrate the mechanism via which clinicopathological features and molecular phenotypes are associated with CTCs and influence clinical outcomes.

\section{Patients and Methods}

Patients and sample collection. Ethics approval (approval number: DYLL2018005) was obtained from the Ethics Committee of Ningbo medical centre Lihuili hospital. A total of 76 patients with CRC diagnosed by colonoscopy and pathological examination at Ningbo Medical Center Lihuili Eastern Hospital from December 2016 to December 2018 were enrolled in this study. There were 55 patients with stage III disease and 21 with stage IV according to the eighth edition of the American Joint Committee on Cancer staging system (8), with 44 males and 32 females, aged from 35 to 82 years old. RAS and $B R A F$ gene mutations were evaluated for all the patients. None of the patients with stage III disease received neoadjuvant radiotherapy and chemotherapy, and CTC detection was performed for all of them prior to surgery. All of the cases with stage IV CRC (17 cases with liver metastases and four cases with lung metastases) were defined as clinically resectable by multi-disciplinary team discussion, and CTC detection was performed before the simultaneous radical resection of metastases and primary foci or neoadjuvant chemotherapy. An oxaliplatin-based perioperative adjuvant chemotherapy regimen of 6 to 8 cycles was administered to the patients.

Clinical information about the patients was gathered, including age, gender, tumor histology, TNM stage, serum carcinoembryonic antigen level, and tumor location. Patients were followed-up for at least 2 years. The primary event monitored was disease-free survival (DFS). Transabdominal enhanced computed tomography and enhanced magnetic resonance imaging were used to determine whether the tumor recurred within 24 months following radical surgery.

Enrichment and detection of CTCs by CellRich ${ }^{T M}$ platform. Peripheral blood samples were collected from 55 patients with stage III CRC before surgery and 21 patients with stage IV CRC before adjuvant platinum-based chemotherapy. CTCs were enriched using the CellRich ${ }^{\mathrm{TM}}$ platform (M\&J Medical, Ningbo, PR China). Using this platform to capture CTCs has been reported elsewhere (9) but using this platform to enrich CTCs has rarely been reported. About $4.0 \mathrm{ml}$ of the patients' peripheral blood was drawn into an acid citrate dextrose anticoagulant tube (Rich Science, Chengdu, PR China) and processed within $48 \mathrm{~h}$. After mixing the contents of the anticoagulant tube by repeated inversion, $4.0 \mathrm{ml}$ of blood was measured with a micropipette, poured into a centrifuge tube and a sample diluent (M\&J Medical) was added to obtain a 45.0-ml solution. After reverse mixing the centrifuge tube, the solution was centrifuged at $700 \times g$ for $5 \mathrm{~min}$ at room temperature. The solution was left to reach $12.0 \mathrm{ml}$ in a vacuum pump (Kylin-Bell, Haimen, PR China), which was mixed with suspension cells and Lysing Solution for Hematology (M\&J Medical) added to bring the volume to $45.0 \mathrm{ml}$. After reverse mixing the centrifuge tube, the solution was placed in a Vertical Mixer (DLAB) at $20 \mathrm{rpm}$ for $10 \mathrm{~min}$ at room temperature and centrifuged at $700 \times g$ for $5 \mathrm{~min}$ at room temperature. The solution above the cell pellet was removed by a vacuum pump. The cells were resuspended and precipitated after adding $5.0 \mathrm{ml}$ Sample Diluent and incubated with Magnetic Particle Supension (M\&J Medical) at $120 \mathrm{rpm}$ for $20 \mathrm{~min}$ at room temperature with Horizantal Rocke (DLAB, Beijing, PR China). The solution was then slowly overlaid onto the Density Reagent (M\&J Medical) and centrifuged at $300 \times g$ for $5 \mathrm{~min}$ at room temperature. The clear solution was removed into a $15.0 \mathrm{ml}$ centrifuge tube which was then placed into the CellRich ${ }^{\mathrm{TM}}$ instrument. After completion of the platform's program, the solution was centrifuged at $2,100 \times g$ for 3 $\mathrm{min}$ at room temperature to collect the cells. Cell suspensions were obtained using CF1 Solution (M\&J Medical) and applied onto slides. The slides were dried at $33^{\circ} \mathrm{C}$ overnight by Dry Cabinet (Yiheng, Shanghai, PR China) for next step.

Immunofluorescence in situ hybridization staining and slide reading. A fluorescence in situ hybridization sample processing kit (M\&J Medical) was used for immunofluorescence in situ hybridization staining. A diluting $1 \times \mathrm{CF} 2$ solution was poured onto slides which were then incubated for $8 \mathrm{~min}$. The slides were then incubated in $2 \times$ saline sodium citrate buffer at $37^{\circ} \mathrm{C}$ for $10 \mathrm{~min}$. Immediately after this, the slides were further incubated in $75 \%, 80 \%$ and $100 \%$ ethanol for $2 \mathrm{~min}$. The Chromosome enumeration probes 8 Orange (CEP8 Orange, M\&J Medical) were titrated onto the slides and they were incubated by the S500 StatSpin ThermoBrite Slide Hybridization/Denaturation System (Abbott, IL, USA). The slides were subsequently incubated in a formamide solution at $43^{\circ} \mathrm{C}$ for $10 \mathrm{~min}$ and then incubated in a $2 \times$ saline sodium citrate buffer at $37^{\circ} \mathrm{C}$ for $5 \mathrm{~min}$. Bovine serum albumin $(0.2 \%)$ diluted in phosphate buffer solution was poured onto the slides, which were then incubated for $3 \mathrm{~min}$. The slides were subjected to immunostaining with Alexa Fluor 594-conjugated monoclonal anti-CD45 for $1 \mathrm{~h}$ in the dark and stained with 4',6-diamidino-2-phenylindole (DAPI). CTCs were identified as DAPI ${ }^{+} / \mathrm{CD} 45^{-}$with aneuploid chromosome 8 by DM3000 (Leica, Wetzlar, Germany).

Tissue immunohistochemical staining. The original hematoxylin and eosin-stained slides were reviewed for each case. Representative areas from the tumor center (composed of intact tumor cells) were marked and embedded in paraffin and cut into $4 \mu \mathrm{m}$ sections. Following deparaffinization, sections were rehydrated and subjected to antigen retrieval by microwaving in $0.01 \mathrm{M}$ sodium citrate $(\mathrm{pH}$ 6) for $10 \mathrm{~min}$. Sections were incubated at $4^{\circ} \mathrm{C}$ overnight with monoclonal antibodies against E-cadherin (Clone 36B5, 1:50 
dilution; Novocastra Laboratories Ltd., Newcastle upon Tyne, UK), and vimentin (Clone V9, 1:100 dilution; Novocastra Laboratories Ltd.). After washing with phosphate-buffered saline, horse-radish peroxidase-conjugated secondary antibody (\#A31460, 1:500; Thermo Fisher Scientific, Inc., Waltham, MA, USA) was added for $60 \mathrm{~min}$ at $37^{\circ} \mathrm{C}$. After several washes with phosphate-buffered saline, staining was achieved using 3,3'-diaminobenzidine for 5-10 min. Finally, the slides were counterstained with Mayer's hemalum and then mounted for microscopy. Protein staining was evaluated under a light microscope at 400x magnification. For E-cadherin, only membranous staining was evaluated, while vimentin (considered a cytoplasmic protein) was evaluated through cytoplasmic staining. Staining intensity was calculated manually by two experienced pathologists. Tumor cells were randomly selected from five fields and scored based on the percentage of positively stained cells $(0-100 \%)$ and a staining intensity score, classified as follows: $0=$ No staining, $1=$ weak staining, $2=$ moderate staining and $3=$ strong staining. The final score was calculated by multiplying the intensity score by the percentage of positively stained cells, resulting in a score ranging between 0 and 3 .

Statistical analysis. The normally distributed categorical data are presented as the mean \pm standard deviation, and intergroup comparisons were performed using the independent $t$-test. Numerical data are expressed as percentages, and intergroup comparisons were conducted with the chi-square test or Fisher's exact test. The risk factors for a high CTC count were subjected to a logistic regression analysis, and odds ratio and $95 \%$ confidence intervals were calculated. Prognostic analyses were performed using Kaplan-Meier survival analysis. All $p$-values were derived using two-sided tests and all of the statistical analyses were conducted using SPSS software version 21.0 (IBM Corp., Armonk, NY, USA) and Prism 5.0 (GraphPad Software Inc., San Diego, CA, USA). A value $p<0.05$ was considered as the threshold for statistical significance.

\section{Results}

Correlation of CTC count with clinicopathological characteristics in patients prior to treatment. The patients were divided into $\mathrm{CTC}^{+}$and $\mathrm{CTC}^{-}$groups according to the results of CTC detection. The $\mathrm{CTC}^{+}$group had CTCs in their peripheral blood while the $\mathrm{CTC}^{-}$group did not. The $\mathrm{CTC}^{+}$ group comprised 32 patients $(42.1 \%)$, and the remaining 44 $(58.9 \%)$ patients were in the $\mathrm{CTC}^{-}$group. There was no statistically significant difference in terms of age distribution, sex, pre-operative carcinoembryonic antigen level, and TNM stage between the $\mathrm{CTC}^{+}$and $\mathrm{CTC}^{-}$groups $(p>0.05)$ (See Table I). Images of captured CTCs from patient 6 is shown in Figure 1.

Evaluation of prognosis of stage III/IV CRC after oxaliplatinbased chemotherapy on the basis of CTC status. Of the 76 patients, seven had missed visits, and the longest follow-up was 24 months. Disease-free survival (DFS) was used as the follow-up index, and the fastest disease progression was 6 months after treatment. At the time of analysis, 20 patients had progressive disease, with 2-year DFS of 73.7\%. Kaplan-
Table I. Correlation between circulating tumor cell (CTC) status and clinicopathological characteristics in patients prior to treatment $(n=76)$.

\begin{tabular}{lccc}
\hline Characteristic & $\mathrm{CTC}^{-}(\mathrm{n}=44)$ & $\mathrm{CTC}^{+}(\mathrm{n}=32)$ & $p$-Value \\
\hline Age, years & & & \\
$\quad$ Mean \pm SD & $65.75 \pm 10.18$ & $65.02 \pm 10.58$ & 0.91 \\
Gender, n (\%) & & & \\
$\quad$ Male & $27(61.4)$ & $16(50)$ & 0.34 \\
Female & $17(38.6)$ & $16(50)$ & \\
T-Stage, n (\%) & & & \\
T1-2 & $2(4.5)$ & $1(3.1)$ & 0.82 \\
T3 & $15(34.1)$ & $13(40.6)$ & \\
T4 & $27(61.4)$ & $18(56.3)$ & \\
N-Stage, n (\%) & $13(29.5)$ & $9(28.1)$ & 0.83 \\
N1 & $26(59.1)$ & $19(59.4)$ & \\
N2 & $5(11.4)$ & $4(12.5)$ & \\
N3 & $37(84.1)$ & $22(68.8)$ & 0.11 \\
M-Stage, n (\%) & $7(15.9)$ & $10(31.2)$ & \\
M0 & & & \\
M1 & $22(50.0)$ & $12(37.5)$ & 0.28 \\
CEA level, n (\%) & $22(50.0)$ & $20(62.5)$ & \\
Normal & & & \\
Abnormal & $11(25)$ & $13(40.6)$ & 0.15 \\
Tumor location, n (\%) & $33(75)$ & $19(59.4)$ & \\
Right & & & \\
Left & & & \\
\hline
\end{tabular}

CEA: Carcinoembryonic antigen.

Meier survival analysis demonstrated that the DFS of the $\mathrm{CTC}^{+}$group was $18.2 \pm 1.3$ weeks (95\% CI=15.6-20.8 weeks), whereas that of the $\mathrm{CTC}^{-}$group was $22.8 \pm 0.5$ weeks $(95 \%$ $\mathrm{CI}=21.8-23.7$ weeks). Furthermore, comparison of the DFS between the $\mathrm{CTC}^{+}$and $\mathrm{CTC}^{-}$groups indicated that the $\mathrm{CTC}^{+}$ group had a worse overall prognosis based on DFS (hazard ratio $=3.58,95 \% \mathrm{CI}=1.414-9.085, p<0.01$; Figure 2).

Correlation between CTC count and histological characteristics. Out of all the 76 patients, $32(42.1 \%)$ and nine $(11.8 \%)$ were identified to have RAS gene mutations and $B R A F$ gene mutations, respectively. A total of 33 patients had mutations in RAS genes, including 27 cases of KRAS mutation and six cases of NRAS mutation. In the $\mathrm{CTC}^{+}$ group, 14 patients $(43.8 \%)$ had $K R A S$ gene mutations, three (9.4\%) had NRAS gene mutations and two (6.3\%) BRAF gene mutations. In the $\mathrm{CTC}^{-}$group, 13 patients $(29.5 \%)$ had KRAS gene mutations, three $(6.8 \%)$ had NRAS gene mutations and seven $(15.9 \%)$ had $B R A F$ gene mutations. However, comparison of the $\mathrm{CTC}^{+}$and $\mathrm{CTC}^{-}$groups in terms of RAS and $B R A F$ mutation rates did not elicit statistically significant differences $(p>0.05)$. E-Cadherin expression in CRC cells was diffuse membranous compared to non-neoplastic colorectal epithelial cells. Vimentin was not expressed in non-neoplastic colorectal epithelial cells. Vimentin was overexpressed in the cytoplasm of the CRC 


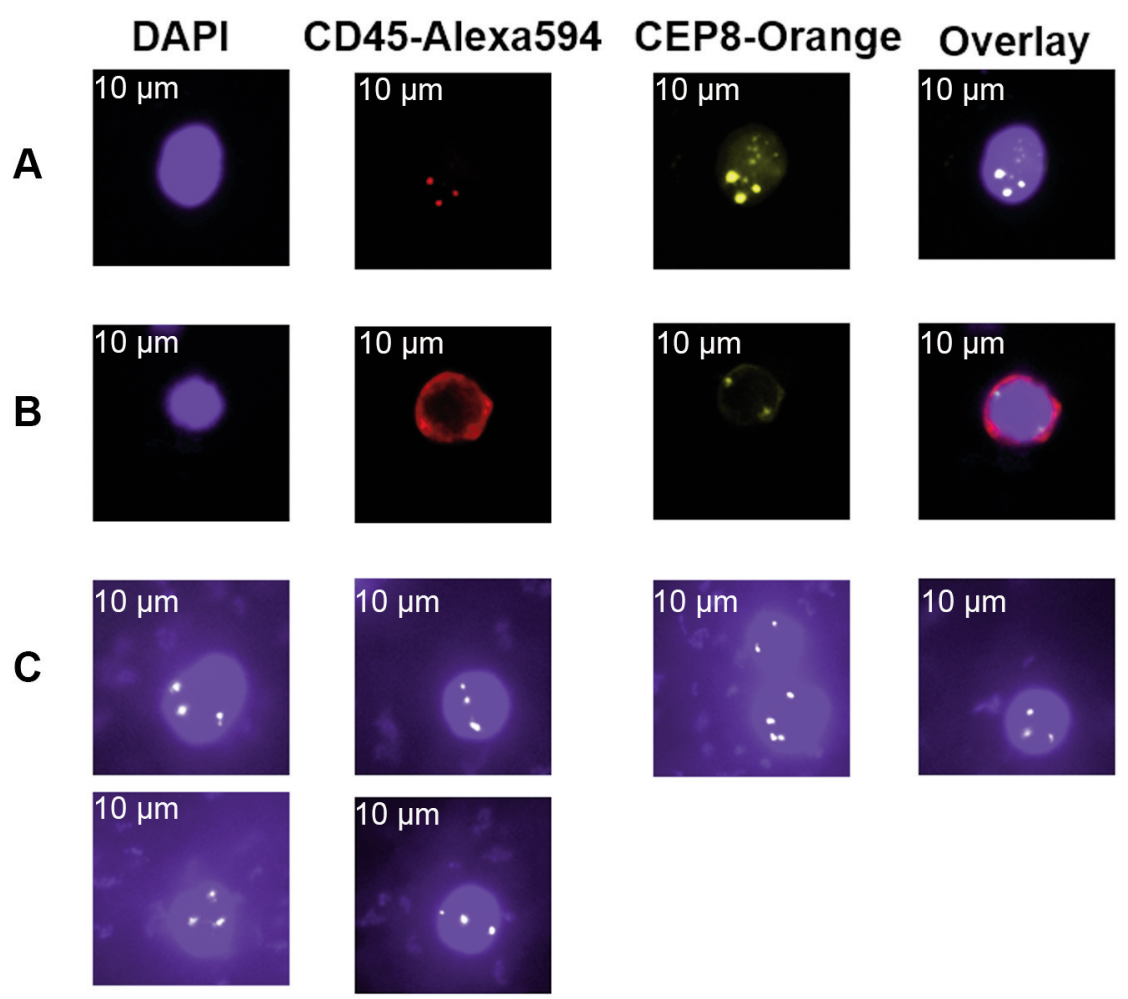

Figure 1. Images of patient's cells as enriched by the CellRich ${ }^{T M}$ System. A: Images of white blood cells (A), cancer cells $(B)$ and cancer cells of patient $6(C)$ as revealed by staining with 4',6-diamidino-2-phenylindole (DAPI), CD45-Alexa594 and CEP8-Orange, and the overlain image.

cells. Compared with the $\mathrm{CTC}^{-}$group, vimentin expression in the $\mathrm{CTC}^{+}$group was significantly increased, while Ecadherin expression was significantly reduced $(p>0.05$, see Figure 3 and Table II).

Univariate analysis and multivariate analysis of the risk factors associated with a high CTC count. Patients with $\geq 3$ CTCs per $4.0 \mathrm{ml}$ of peripheral blood were classified into the group with a high CTC count, whereas those with $<3$ CTCs were classified as having a low CTC count. Univariate analysis showed that among the clinicopathological factors, tumor location in the right colon (ascending colon) and clinically resectable distant metastasis were risk factors associated with a high CTC count. In terms of histological and molecular factors, we found that the group with a high CTC count comprised 11 patients (64.7\%) with KRAS mutations and 14 patients $(82.4 \%)$ with RAS mutations. This group had a higher rate of KRAS and RAS mutations compared to the group with a low CTC count but the difference between these two groups in terms of NRAS and $B R A F$ mutation rates was not statistically significant $(p>0.05)$. Vimentin expression in the group with a high CTC count was still significantly elevated, while the expression of E-cadherin was considerably reduced $(p>0.05$, see Table

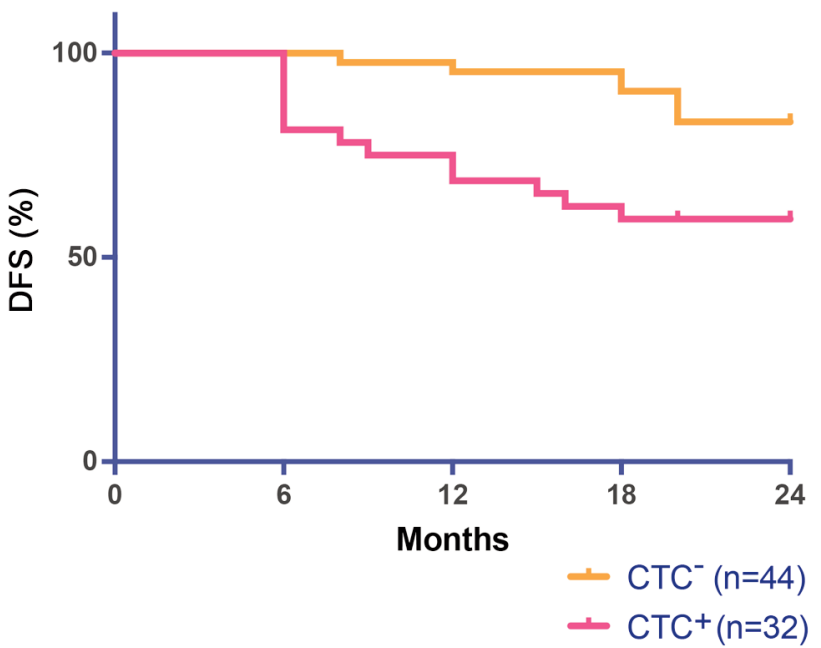

Figure 2. Kaplan-Meier curve of disease-free survival according to circulating tumor cell (CTC) status at primary diagnosis.

III, Figure 3). Logistic multivariate analysis confirmed that RAS gene mutation and high vimentin expression were independent risk factors associated with a high CTC count $(p>0.05$, see Table IV). 



Figure 3. Immunohistochemical analysis of the expression of the epithelial-mesenchymal transition-related proteins vimentin (A) and E-cadherin (B) in non-neoplastic tissue $(\times 400)$ and tumor $(\times 200)$.

\section{Discussion}

CTCs are cancer cells found in the peripheral blood that originate from either the primary tumor or its metastases, and thus possess the primary tumor's genetic and epigenetic characteristics $(10,11)$. With the maturation of technology through improvement of its sensitivity and specificity, CTC detection has become a useful and non-invasive diagnostic tool. In the present investigation, CTCs were enriched using the CellRich ${ }^{\mathrm{TM}}$ platform which has been previously reported for capturing CTCs (9). This detection system is composed of an immunomagnetic particle capture and matching kit. Microfluidic chip technology and gradient magnetic fields are used to specifically adsorb the immunomagnetic particle complex (cell), achieving the capture/enrichment of CTCs.
The CTCs were then stained for identification. Previously published studies have proven that this system can effectively detect the number of CTCs in the peripheral blood of patients with tumor with good sensitivity and specificity. In the present study, we investigated the relationship between CTC count and the clinicopathological characteristics in patients with advanced CRC. Firstly, 55 patients with stage III and 21 patients with stage IV CRC were enrolled. On further analysis, 32 patients were determined as being $\mathrm{CTC}^{+}$at the preoperative or prechemotherapy baseline examination. The $\mathrm{CTC}^{+}$rate was as high as $42.1 \%$, consistent with the data reported by other studies (10-12). This shows that patients with advanced CRC are susceptible to hematogenous spread and metastasis of tumor cells, therefore adequate adjuvant chemotherapy is 
Table II. Correlation between the circulating tumor cell (CTC) count and histological characteristics in patients prior to treatment $(n=76)$.

\begin{tabular}{lccc}
\hline Characteristic & $\mathrm{CTC}^{-}(\mathrm{n}=44)$ & $\mathrm{CTC}^{+}(\mathrm{n}=32)$ & $p$-Value \\
\hline $\begin{array}{l}\text { KRAS, n (\%) } \\
\text { Wild-type }\end{array}$ & $26(59.1)$ & $14(43.8)$ & 0.19 \\
$\quad$ Mutated & $18(40.9)$ & $18(56.2)$ & \\
$\begin{array}{l}\text { NRAS, n (\%) } \\
\text { Wild-type }\end{array}$ & $41(93.2)$ & $29(90.6)$ & 0.68 \\
$\quad$ Mutated & $3(6.8)$ & $3(9.4)$ & \\
$\begin{array}{l}\text { BRAF, n (\%) } \\
\text { Wild-type }\end{array}$ & $38(86.4)$ & $24(75.0)$ & 0.21 \\
$\quad$ Mutated & $6(13.6)$ & $8(25.0)$ & \\
$\begin{array}{l}\text { Vimentin IHC score } \\
\quad \text { Mean } \pm \text { SD }\end{array}$ & $1.17 \pm 0.57$ & $1.52 \pm 0.45$ & $<0.01$ \\
E-Cadherin IHC score & & & \\
$\quad$ Mean \pm SD & $0.87 \pm 0.58$ & $1.21 \pm 0.65$ & 0.02 \\
\hline
\end{tabular}

IHC: Immunohistochemistry.

essential. Nevertheless, up to $20-30 \%$ of patients with advanced CRC experienced disease progression even after administration of adjuvant chemotherapy in strict accordance with the guidelines (13), which manifested as recurrence and metastasis after radical surgery, or disease progression during neoadjuvant treatment. In this study, after a 2-year followup, $28.9 \%$ of patients had disease progression, and the 2-year DFS was $71.1 \%$, which is consistent with the data reported by other studies (14). Further, we found that the $\mathrm{CTC}^{+}$and $\mathrm{CTC}^{-}$groups had similar clinical and pathological characteristics at baseline, and that prognostic differences were evident between the two groups after first-line adjuvant chemotherapy based on oxaliplatin. The $\mathrm{CTC}^{+}$group had earlier recurrence and metastasis after radical surgery or disease progression during neoadjuvant therapy. Therefore, we believe that the CTC count can be used as a predictor of the efficacy of oxaliplatin-based first-line chemotherapy for stage III and IV CRC. Further research is required to determine whether it is necessary to change the first-line chemotherapy regimen or whether additional chemotherapy is needed after full-course chemotherapy.

In order to further clarify the mechanism through which clinicopathological features and molecular phenotypes are associated with the CTC count, we conducted univariate and logistic regression analyses. The results suggested that RAS gene mutations and high vimentin expression were the independent risk factors associated with an increased CTC count.

RAS and BRAF genotypic status are indicators of tumor biological characteristics in CRC. RAS mutations suggest tumor resistance to targeted therapy with cetuximab. CTCs are viewed as a good source of DNA and RNA for analyses. Analysis of RAS genotype status directly from CTCs may be of great clinical importance. However, the DNA obtained using the
Table III. Univariate analysis of risk factors associated with a circulating tumor cell count of 3 or higher per $4.0 \mathrm{ml}(\mathrm{CTCh}+)$.

\begin{tabular}{|c|c|c|c|}
\hline Characteristic & $\mathrm{CTC}^{\mathrm{h}-}(\mathrm{n}=59)$ & $\mathrm{CTC}^{\mathrm{h}+}(\mathrm{n}=17)$ & $p$-Value \\
\hline \multicolumn{4}{|l|}{ Age, years } \\
\hline Mean \pm SD & $64.64 \pm 10.22$ & $64.52 \pm 11.30$ & 0.96 \\
\hline \multicolumn{4}{|l|}{ Gender, n (\%) } \\
\hline Male & $25(42.4)$ & $9(52.9)$ & \multirow[t]{2}{*}{0.44} \\
\hline Female & $34(57.6)$ & $8(47.1)$ & \\
\hline \multicolumn{4}{|l|}{ T-Stage, $\mathrm{n}(\%)$} \\
\hline $\mathrm{T} 1-2$ & $2(3.4)$ & $1(5.9)$ & \multirow[t]{3}{*}{0.72} \\
\hline $\mathrm{T} 3$ & $23(40.0)$ & $5(29.4)$ & \\
\hline $\mathrm{T} 4$ & $34(56.6)$ & $11(64.7)$ & \\
\hline \multicolumn{4}{|l|}{ N-Stage, n (\%) } \\
\hline N1 & $19(32.2)$ & $3(17.6)$ & \multirow[t]{3}{*}{0.17} \\
\hline $\mathrm{N} 2$ & $33(55.9)$ & $9(82.4)$ & \\
\hline N3 & 7 (11.9) & $5(29.4)$ & \\
\hline \multicolumn{4}{|l|}{ M-Stage, n (\%) } \\
\hline M0 & $49(83.1)$ & $10(70.6)$ & \multirow[t]{2}{*}{0.04} \\
\hline M1 & $10(16.9)$ & $7(29.4)$ & \\
\hline \multicolumn{4}{|c|}{ CEA level, n (\%) } \\
\hline Normal & $29(49.2)$ & $5(29.4)$ & \multirow[t]{2}{*}{0.15} \\
\hline Abnormal & $30(50.8)$ & $12(70.6)$ & \\
\hline \multicolumn{4}{|l|}{ Tumor location } \\
\hline Right & $14(23.7)$ & $9(52.9)$ & \multirow[t]{2}{*}{0.03} \\
\hline Left & $45(76.3)$ & $8(47.1)$ & \\
\hline \multicolumn{4}{|l|}{$K R A S, \mathrm{n}(\%)$} \\
\hline Wild-type & $36(60.0)$ & $4(23.5)$ & \multirow[t]{2}{*}{$<0.01$} \\
\hline Mutated & $23(40.0)$ & $13(76.5)$ & \\
\hline \multicolumn{4}{|l|}{$N R A S, \mathrm{n}(\%)$} \\
\hline Wild-type & $56(94.9)$ & $14(82.4)$ & \multirow[t]{2}{*}{0.09} \\
\hline Mutated & $3(5.1)$ & $3(17.6)$ & \\
\hline \multicolumn{4}{|l|}{$B R A F, \mathrm{n}(\%)$} \\
\hline Wild-type & $49(83.1)$ & $13(76.5)$ & \multirow[t]{2}{*}{0.54} \\
\hline Mutated & $10(16.9)$ & $4(23.5)$ & \\
\hline \multicolumn{4}{|l|}{ RAS } \\
\hline Wild-type & $34(57.6)$ & $2(11.8)$ & \multirow[t]{2}{*}{$<0.01$} \\
\hline Mutated & $25(42.4)$ & $15(88.2)$ & \\
\hline \multicolumn{4}{|c|}{ Vimentin IHC score } \\
\hline Mean \pm SD & $1.20 \pm 0.54$ & $1.72 \pm 0.35$ & $<0.01$ \\
\hline \multicolumn{4}{|c|}{ E-cadherin IHC score } \\
\hline Mean+SD & $1.17 \pm 0.62$ & $0.70 \pm 0.57$ & $<0.01$ \\
\hline
\end{tabular}

CEA: Carcinoembryonic antigen; IHC: immunohistochemistry.

CellSearch $^{\mathrm{TM}}$ system was not suitable for KRAS analysis (12). In this study, RAS genotype was directly examined in tissue samples and its relationship to CTCs was analyzed. We found 33 cases of RAS mutations, with a mutation rate of $43.4 \%$, with the majority being KRAS mutations $(\mathrm{n}=27)$. There was no statistical difference between the $\mathrm{CTC}^{+}$and the $\mathrm{CTC}^{-}$groups in terms of KRAS, NRAS and BRAF mutation rates. Interestingly, in accordance with other studies $(10,15)$, CTC counts of 3 or more in $7.5 \mathrm{ml}$ of peripheral blood was defined as a high CTC count, and we found that in 17 patients with a high CTC count the KRAS mutation rate was $64.7 \%$ and the RAS mutation rate was $82.7 \%$, rates which were significantly 
Yu et al: CTC in Colorectal Cancer Chemotherapy

Table IV. Logistic multivariate analysis of risk factors associated with a circulating tumor cell count of 3 or higher per $4.0 \mathrm{ml}\left(C T C^{h+}\right)$.

\begin{tabular}{lccccccc}
\hline Factor $^{\mathrm{a}}$ & $\mathrm{B}$ & Std. error & Wald & df & $p$-Value & Exp (B) & $95 \%$ CI for Exp (B) \\
\hline Vimentin & 2.154 & 1.046 & 4.239 & 1 & 0.039 & 8.621 & $1.109-67.016$ \\
E-Cadherin & -1.123 & 0.658 & 2.912 & 1 & 0.088 & 0.325 & $0.089-1.182$ \\
M0 stage & -0.524 & 0.780 & 0.452 & 1 & 0.501 & 0.592 & $0.128-2.731$ \\
M1 stage & $0^{\mathrm{b}}$ & & & 0 & & - \\
$R A S$ wild-type & -1.907 & 0.876 & 4.742 & 1 & 0.029 & 0.149 & $0.027-0.826$ \\
$R A S$ mutated & $0^{\mathrm{b}}$ & 0. & - & 0 & & 0.0 .5 & $0.113-2.945$ \\
Tumor location, left & -0.550 & 0.832 & 0.437 & 1 & 0.509 & 0.577 & \\
Tumor location, right & $0^{\mathrm{b}}$ & 0 & & 0 & & & \\
\hline
\end{tabular}

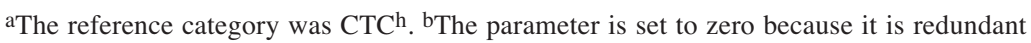

higher than the group with a low CTC count. These findings also suggest that targeted therapy with cetuximab may have worse efficacy in patients with a high CTC count, which needs to be confirmed by further studies.

EMT is an important process in the metastatic cascade, which significantly improves the ability of tumor cells to invade and metastasize (16). Chebouti et al. found that EMT-like CTCs were more abundant than epithelial CTCs in patients with ovarian cancer (17). This finding is in accordance with results obtained in breast cancer, in a study reporting that EMT is a rare event in the primary tumor but frequently occurs in CTCs (18, 19). Based on these facts, we firmly believe that EMT in primary CRC may be an important etiological factor for the formation of CTCs. Vimentin is regarded as a sign of cell epithelial to mesenchymal conversion and seems to be one of the best indicators of EMT in tumorigenesis (20). An increasing number of studies have investigated the prognostic roles of vimentin expression and its clinicopathological significance in cancer (21-23). However, the results of the published studies were inconsistent. The contradictions between published studies may result from the differences in sample size, CRC stage, molecular pathology and the study design. Furthermore, other markers may also influence the progression and prognosis of cancer through the regulation of vimentin expression. To the best of our knowledge, this report is the first study combining univariate and multivariate analyses showing that high vimentin expression is an independent risk factor for CTC formation. It is possible that tumor EMT may have resulted in an increased number of CTCs and ultimately a worsening of prognosis.

In conclusion, this is the first study demonstrating that CTC count in CRC can be used as a predictor of the efficacy of platinum-based first-line chemotherapy. We therefore encourage further investigation of risk factors which might be associated with a high CTC count. We believe that through this study, there are two points worth pondering: i) RAS mutations in the primary tumor and high expression of the EMT marker vimentin are independent risk factors associated with a high CTC count. RAS genotypic status is currently included in the clinical routine. The biomarkers of EMT can be obtained from postoperative tissue samples. An immunohistochemical test can be performed to determine whether these two indicators can be used to predict the efficacy of oxaliplatin-based chemotherapy where technology for determining CTCs is not available or not used as a routine test method; ii) It needs to be determined whether or not it is necessary to change the first-line chemotherapy regimen to improve the prognosis of patients with a positive CTC status, which requires further large-sample clinical randomized controlled studies.

\section{Conflicts of Interest}

The Authors have no conflicts of interest, financial or otherwise.

\section{Authors' Contributions}

Study conception and design: Mian Yang andJohn Zhang. Acquisition of data: Jiazi Yu, John Zhang, Tao Peng, Zhenglei Fei and Liangbin Jin. Analysis and interpretation of data: Mian Yang and Jiazi Yu. Drafting of article: Jiazi Yu.

\section{References}

1 Bray F, Ferlay J, Soerjomataram I, Siegel RL, Torre LA and Jemal A: Global cancer statistics 2018: GLOBOCAN estimates of incidence and mortality worldwide for 36 cancers in 185 countries. CA Cancer J Clin 68(6): 394-424, 2018. PMID: 30207593. DOI: $10.3322 /$ caac. 21492

2 Yoshino T, Arnold D, Taniguchi H, Pentheroudakis G, Yamazaki K, Xu RH, Kim TW, Ismail F, Tan IB, Yeh KH, Grothey A, Zhang S, Ahn JB, Mastura MY, Chong D, Chen LT, Kopetz S, EguchiNakajima T, Ebi H, Ohtsu A, Cervantes A, Muro K, Tabernero J, Minami H, Ciardiello F and Douillard JY: Pan-Asian adapted ESMO consensus guidelines for the management of patients with metastatic colorectal cancer: a JSMO-ESMO initiative endorsed by CSCO, KACO, MOS, SSO and TOS. Ann Oncol 29(1): 44-70, 2018. PMID: 29155929. DOI: $10.1093 /$ annonc/mdx 738

3 Alix-Panabières $\mathrm{C}$ and Pantel $\mathrm{K}$ : Clinical applications of circulating tumor cells and circulating tumor DNA as liquid biopsy. Cancer Discov 6(5): 479-491, 2016. PMID: 26969689. DOI: $10.1158 / 2159-8290 . C D-15-1483$ 
4 Diaz LA Jr and Bardelli A: Liquid biopsies: genotyping circulating tumor DNA. J Clin Oncol 32(6): 579-586, 2014. PMID: 24449238. DOI: 10.1200/JCO.2012.45.2011

5 Chaffer CL and Weinberg RA: A perspective on cancer cell metastasis. Science 331(6024): 1559-1564, 2011. PMID: 21436443. DOI: $10.1126 /$ science. 1203543

6 Mego M, Mani SA, Lee BN, Li C, Evans KW, Cohen EN, Gao H, Jackson SA, Giordano A, Hortobagyi GN, Cristofanilli M, Lucci A and Reuben JM: Expression of epithelial-mesenchymal transition-inducing transcription factors in primary breast cancer: The effect of neoadjuvant therapy. Int J Cancer 130(4): 808-816, 2012. PMID: 21387303. DOI: 10.1002/ijc.26037

7 Yang J, Ma J, Jin Y, Cheng S, Huang S, Zhang N and Wang Y: Development and validation for prognostic nomogram of epithelial ovarian cancer recurrence based on circulating tumor cells and epithelial-mesenchymal transition. Sci Rep 11(1): 6540, 2021. PMID: 33753862. DOI: 10.1038/s41598021-86122-4

8 Weiser MR: AJCC 8th edition: Colorectal cancer. Ann Surg Oncol 25(6): 1454-1455, 2018. PMID: 29616422. DOI: 10.1245/s10434-018-6462-1

9 Shen W, Song Y, Burklund A, Le B, Zhang R, Wang L, Xi Y, Qian K, Shen T and Zhang JXJ: Combined immunomagnetic capture coupled with ultrasensitive plasmonic detection of circulating tumor cells in blood. Biomed Microdevices 20(4): 99, 2018. PMID: 30417219. DOI: 10.1007/s10544-018-0333-1

10 Bidard FC, Kiavue N, Ychou M, Cabel L, Stern MH, Madic J, Saliou A, Rampanou A, Decraene C, Bouché O, Rivoire M, Ghiringhelli F, Francois E, Guimbaud R, Mineur L, KhemissaAkouz F, Mazard T, Moussata D, Proudhon C, Pierga JY, Stanbury T, Thézenas S and Mariani P: Circulating tumor cells and circulating tumor DNA detection in potentially resectable metastatic colorectal cancer: a prospective ancillary study to the Unicancer Prodige-14 trial. Cells 8(6): 516, 2019. PMID: 31142037. DOI: 10.3390/cells8060516

11 Tol J, Koopman M, Miller MC, Tibbe A, Cats A, Creemers GJ, Vos AH, Nagtegaal ID, Terstappen LW and Punt CJ: Circulating tumour cells early predict progression-free and overall survival in advanced colorectal cancer patients treated with chemotherapy and targeted agents. Ann Oncol 21(5): 1006-1012, 2010. PMID: 19861577. DOI: $10.1093 /$ annonc/mdp463

12 Otsuka K, Imai H, Soeda H, Komine K, Ishioka C and Shibata $\mathrm{H}$ : Practical utility of circulating tumour cells as biomarkers in cancer chemotherapy for advanced colorectal cancer. Anticancer Res 33(2): 625-629, 2013. PMID: 23393358.

13 Cohen SJ, Punt CJ, Iannotti N, Saidman BH, Sabbath KD, Gabrail NY, Picus J, Morse MA, Mitchell E, Miller MC, Doyle GV, Tissing H, Terstappen LW and Meropol NJ: Prognostic significance of circulating tumor cells in patients with metastatic colorectal cancer. Ann Oncol 20(7): 1223-1229, 2009. PMID: 19282466. DOI: $10.1093 /$ annonc/mdn786

14 Zhao R, Cai Z, Li S, Cheng Y, Gao H, Liu F, Wu S, Liu S, Dong Y, Zheng L, Zhang W, Wu X and Yao X: Expression and clinical relevance of epithelial and mesenchymal markers in circulating tumor cells from colorectal cancer. Oncotarget 8(6): 9293-9302, 2017. PMID: 28030836. DOI: 10.18632/oncotarget.14065

15 Cohen SJ, Punt CJ, Iannotti N, Saidman BH, Sabbath KD, Gabrail NY, Picus J, Morse M, Mitchell E, Miller MC, Doyle GV, Tissing H, Terstappen LW and Meropol NJ: Relationship of circulating tumor cells to tumor response, progression-free survival, and overall survival in patients with metastatic colorectal cancer. J Clin Oncol 26(19): 3213-3221, 2008. PMID: 18591556. DOI: $10.1200 / \mathrm{JCO} .2007 .15 .8923$

16 Zheng X, Carstens JL, Kim J, Scheible M, Kaye J, Sugimoto H, Wu CC, LeBleu VS and Kalluri R: Epithelial-to-mesenchymal transition is dispensable for metastasis but induces chemoresistance in pancreatic cancer. Nature 527(7579): 525530, 2015. PMID: 26560028. DOI: 10.1038/nature16064

17 Chebouti I, Kasimir-Bauer S, Buderath P, Wimberger P, Hauch S, Kimmig R and Kuhlmann JD: EMT-like circulating tumor cells in ovarian cancer patients are enriched by platinum-based chemotherapy. Oncotarget 8(30): 48820-48831, 2017. PMID: 28415744. DOI: 10.18632/oncotarget.16179

18 Yu M, Bardia A, Wittner BS, Stott SL, Smas ME, Ting DT, Isakoff SJ, Ciciliano JC, Wells MN, Shah AM, Concannon KF, Donaldson MC, Sequist LV, Brachtel E, Sgroi D, Baselga J, Ramaswamy S, Toner M, Haber DA and Maheswaran S: Circulating breast tumor cells exhibit dynamic changes in epithelial and mesenchymal composition. Science 339(6119): 580584, 2013. PMID: 23372014. DOI: 10.1126/science.1228522

19 Kasimir-Bauer S, Hoffmann O, Wallwiener D, Kimmig R and Fehm T: Expression of stem cell and epithelial-mesenchymal transition markers in primary breast cancer patients with circulating tumor cells. Breast Cancer Res 14(1): R15, 2012. PMID: 22264265. DOI: 10.1186/bcr3099

20 Fan F, Samuel S, Evans KW, Lu J, Xia L, Zhou Y, Sceusi E, Tozzi F, Ye XC, Mani SA and Ellis LM: Overexpression of snail induces epithelial-mesenchymal transition and a cancer stem cell-like phenotype in human colorectal cancer cells. Cancer Med 1(1): 5-16, 2012. PMID: 23342249. DOI: 10.1002/cam4.4

21 Liu M, Wang R, Sun X, Liu Y, Wang Z, Yan J, Kong X, Liang S, Liu Q, Zhao T, Ji X, Wang G, Wang F, Wang G, Chen L, Zhang Q, Lv W, Li H and Sun M: Prognostic significance of PD-L1 expression on cell-surface vimentin-positive circulating tumor cells in gastric cancer patients. Mol Oncol 14(4): 865-881, 2020. PMID: 31981446. DOI: 10.1002/1878-0261.12643

22 Jin H, Morohashi S, Sato F, Kudo Y, Akasaka H, Tsutsumi S, Ogasawara H, Miyamoto K, Wajima N, Kawasaki H, Hakamada $\mathrm{K}$ and Kijima H: Vimentin expression of esophageal squamous cell carcinoma and its aggressive potential for lymph node metastasis. Biomed Res 31(2): 105-112, 2010. PMID: 20460738. DOI: $10.2220 /$ biomedres.31.105

23 Overman MJ, Morris V, Moinova H, Manyam G, Ensor J, Lee MS, Eng C, Kee B, Fogelman D, Shroff RT, LaFramboise T, Mazard T, Feng T, Hamilton S, Broom B, Lutterbaugh J, Issa JP, Markowitz SD and Kopetz S: Phase I/II study of azacitidine and capecitabine/oxaliplatin (CAPOX) in refractory CIMP-high metastatic colorectal cancer: evaluation of circulating methylated vimentin. Oncotarget 7(41): 67495-67506, 2016. PMID: 27542211. DOI: $10.18632 /$ oncotarget.11317 